\title{
INTRODUCTION TO COASTAL HF MARITIME SURVEILLANCE RADARS
}

\begin{abstract}
This paper presents the main technical characteristics and working performances of coastal maritime surveillance radars, such as low-power High-Frequency Surface Wave Radars (HFSWR) and Over the Horizon Radars (OTHR). These radars have demonstrated to be a cost-effective long-range early-warning sensor for ship detection and tracking in coastal waters, sea channels and passages. In this work, multi-target tracking and data fusion techniques are applied to live-recorded data from a network of oceanographic HFSWR stations installed in Jindalee Operational Radar Network (JORN), Wellen Radar (WERA) in Ligurian Sea (Mediterranean Sea), CODAR Ocean Sebsorsin and in the German Bight (North Sea). The coastal Imaging Sciences Research (ISR) HFSWR system, Multi-static ISR HF Radar, Ship Classification using Multi-Frequency HF Radar, Coastal HF radar surveillance of pirate boats and Different projects of coastal $\mathrm{HF}$ radars for vessels detecting are described.

Ship reports from the Automatic Identification System (AIS), recorded from both coastal and satellite Land Earth Stations (LES) are exploited as ground truth information and a methodology is applied to classify the fused tracks and to estimate system performances. Experimental results for all above solutions are presented and discussed, together with an outline for future integration and infrastructures.
\end{abstract}

Keywords: HFSWR, OTHR, JORN, WERA, ISR, AIS, LES, LPA, LRA, DOA, DDC, PCI, RCS

\section{INTRODUCTION}

Over the years there have been many systems developed to serve coastal HF maritime surveillance radars, but more known are HFSWR and OTHR. They use the frequency band of 3-30 MHz to provide a large coverage that could extend up to $200 \mathrm{NM}$ in range. Thus, the primary benefits of the HF-band for coastal radars are over the horizon capability, sky-wave and groundwave propagation modes, which simplified signal generation and processing requirements. Traditional system design for coastal surveillance radars includes large bistatic HF transmit and receive arrays with a substantial geographic footprint since a wide aperture array is used to provide the needed resolution for detecting surface targets while reducing clutter. Although proven to be effective, the infrastructure requirements of these systems generally render them large, quite costly and not environmentally friendly and immobile, creating major limitations for wide deployment. However, the increased emphasis on homeland security applications such as maritime surveillance for enhanced safety at sea, national security and enforcement continues to drive the need for efficient mobile and low-cost HFSWR and OTHR systems. In order to meet the demands of homeland security applications while keeping the costs, environmental impact and infrastructure requirements at a minimum, novel integrated HF radar system designs are required. 


\section{COASTAL ISR HFSWR SYSTEM}

The costal HFSWR and OTHR radars are a highly costeffective remote sensing technology for measuring waves, swell direction and for monitoring the movements and detecting of ships and aircraft at over the horizon ranges including pirate boats. The US Imaging Sciences Research (ISR) company is currently developing a low cost, compact HF Phase Array Radar to measure directional wave spectra along a single radial line offshore, without requiring beam steering. The project of ISR is being funded for this work as a Bureau of Ocean Energy Management by NOAA National Oceanographic Partnership Program (NOPP) participant.

However, a bistatic HF radar has been developed for application to ocean current mapping and especially for ship vector tracking. The radar can operate in a multi-frequency mode, so that it can map ocean current vertical shear and can provide more robust ship tracks than single frequency HF radars. This tracking robustness is achieved by avoiding target fading due to echo nulls from frequency and azimuth variations in ship radar cross-section that occur using a single radar frequency. The radar is fully digital in frequency generation and reception and has no RF receiver components because the received antenna signals are digitized at the HF frequency directly. The system uses Analogue-to-Digital (A/D) conversion rates sufficiently high to maintain the 2 to 1 frequency ratio required for the highest radar frequency of interest to avoid frequency aliasing.

The newly developed radar acquisition code provides realtime range compression so that data files that are stored in the processor are In-phase and Quadrature (I/Q) samples, at a much less dense rate than the original digitized signal time series. Thus, the bistatic capability is based on accurate system timing and radar frequency. These are provided at each of two or more radar sites by rubidium clocks and GPS timing, accurate for the first pulse to 50-ns to initiate data acquisition in the bistatic mode. Once acquisition is initiated, the rubidium clocks at each site maintain much more accurate frequency and time stability to allow Doppler velocity measurements accurate to $2 \mu \mathrm{Hz}$ at $25 \mathrm{MHz}$ operating frequency.

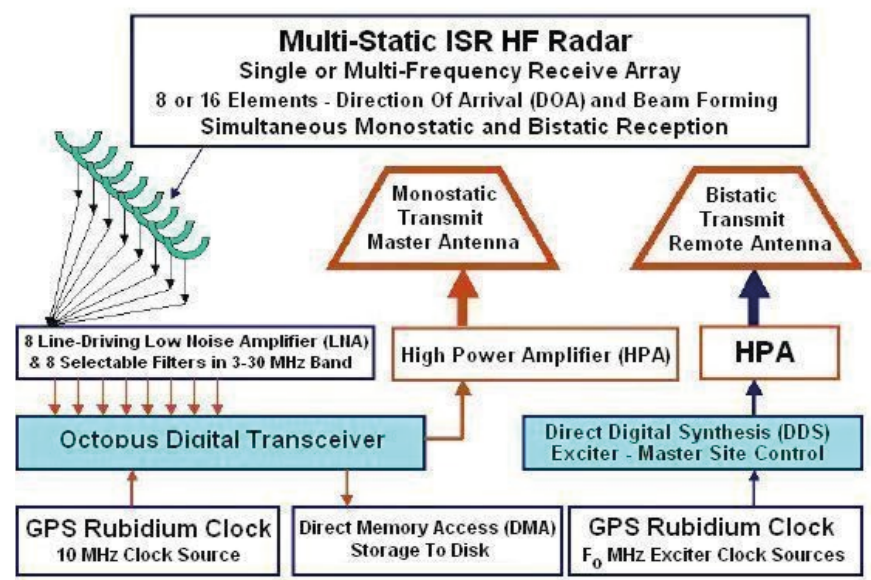

Fig. 1. System Layout of Typical Bistatic Two-site HF Radar-Courtesy of Manual: by ISR [1]
The primary site requires an 8 or 16-element receives array, and both primary and satellite bistatic-illuminator sites have a modest 2 or 4 -element monopole transmit antenna pair. This bistatic approach reduces the coastal space requirements because of the need for just one receive-antenna array per radar system.

These systems can be operated with a pair of bistatic transmitters, either side of the receive site, to expand the spatial coverage. Using such an approach, these units could be staggered to create a system of few radars, providing continuous coverage along a coastline, alternating transmit and receive sites. This type of arrangement could be used to provide robust ship tracking along a country's coastline, and a modest estimate of type and tonnage of all vessel traffic based on target echo strength. Due to its digital approach, the cost of these radars is substantially lower than that of existing single coastal HF radars, none of which have multifrequency capability $[1,2,3,4]$.

\section{MULTI-STATIC ISR HF RADAR}

The ISR Company has developed a bistatic HF radar for robust ship tracking in coastal seas and ocean current mapping based on purely digital receiver and transmission technology. The bistatic capability represents an upgrade from the previous system, requires use of accurate system clocks and absolute time referencing in order to operate in a bistatic mode. These are provided by GPS timing and rubidium clocks. Initially, the system was comprehensively tested in the VHF-band and some of those results are presented here. The VHF-band allows for shorter range and wider bandwidth usage than can be achieved at HF frequency. The technology transfers directly to HF frequencies, with slower A/D conversion rates required due to lower radar frequencies and corresponding lower Nyquist frequencies, named after engineer Harry Nyquist, as $1 / 2$ of the sampling rate of a discrete signal processing system.

A layout of the bistatic radar is shown in Figure $\mathbf{1}$ for a 2-site system, with the master site on the left and a bistatic transmitter shown on the right. The heart of the radar is the Octopus transceiver card, which has both a programmable pulsing capability and 8-receive channels per card. For a 16-element array, a second Octopus Receiver card provides eight additional channels of receives capability. At the bistatic transmit-only site, an Exciter card is used to generate the pulsed waveforms.

Typically, the radar is operated with up to a $20 \%$ duty cycle pulse compressed waveform, fully programmable by the operator. For long-range operation, for example, a $100 \mu \mathrm{s}$ pulse is transmitted (forcing a $15 \mathrm{Km}$ blind area around the area), which is compressed to $10 \mu \mathrm{s}$, achieving a $20 \mathrm{~dB}$ pulse compression gain. Thus, this allows a $250 \mathrm{~W}$ peak power pulse to be compressed to the equivalent of a $5 \mathrm{KW}$ pulse using frequency-modulated pulse waveforms.

The receive array at the master site receives both monostatic echoes from pulses transmitted at the master site, as well 
as bistatic echoes from pulses transmitted at the slave site. The pulses can either be interleaved on ping-pong like time sequence or can be transmitted simultaneously at two different radar frequencies with non-overlapping frequency content.

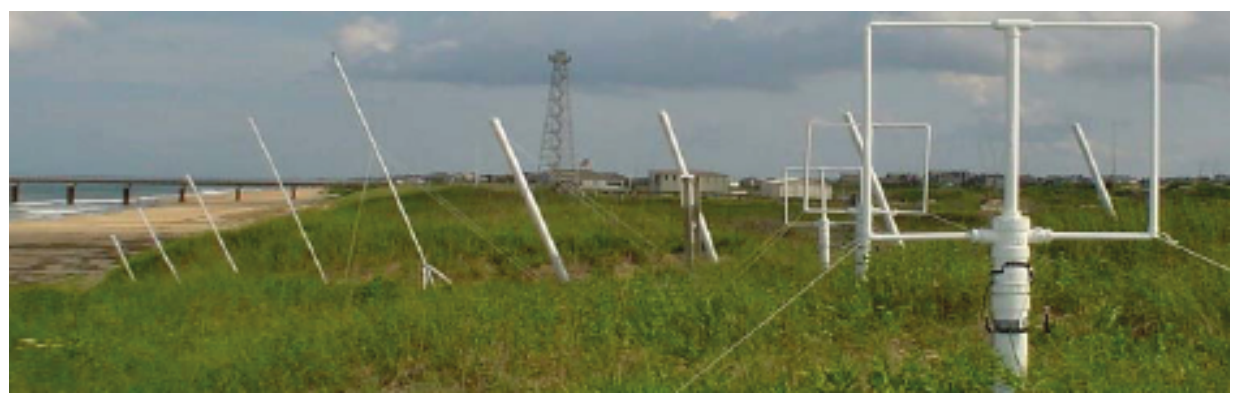

Fig. 2. Multi-Frequency Radar Transmit LPA and Receive LRACourtesy of Paper: by Trizna [5]

Additionally, one array could use orthogonal phase modulated waveforms simultaneously for optimal use of frequency-time bandwidth. In the case of multi-frequency operation, more complicated antennas at both, transmit and receive end, which must be used to accommodate the desired bandwidth. It is developing a low-cost single-frequency system that will use off the shelf antenna components to minimize cost and space requirements. A multi-frequency system covering the full 3-30 MHz HF band requires wideband antennas and switchable narrowband filters to assure good signal to noise ratios for reliable ship tracking. The long-periodic array is most suitable for such a wide operating bandwidth. Operation over a smaller fraction of the HF band, as might be required for long-range application in order to minimize radar propagation losses in the surface wave mode, might, for example, require only a 3-10 $\mathrm{MHz}$ bandwidth. One can achieve this by using transmit antennas in a short two or four element transmit array, with trap antennas or multi-mode elements with several resonances that have low reflection and good standing wave ratios at several frequencies in the desired band. For receiving antennas for multi-frequency radar, loop antennas provide good bandwidth and some directivity to minimize reception of the transmitted pulse.

A new transmit radar antenna was designed for broadband operation over the entire 3-30 MHz band, a modified Log Periodic Antenna (LPA), many of which are shown in Figure 2. However, more compact designs are feasible for operation at pre-specified selected frequencies of four or eight in number, e.g. for operational current shear maps. The LPA antenna provides full tunability to any frequency in the HF band and is suggested for target classification applications, such as small and large ships or current shear experiments.

The receive Loop Receive Array (LRA) antenna is based on a loop design, three of which are shown in Figure 2. The ISR internal broad preamplifiers provide impedance matching to the loop over the 3-30 MHz filtered output. These elements can be arranged for Direction-of-Arrival (DOA) processing. A small 4-element loop array requires less space than long linear arrays, and is useful for current mapping and ship classification, but could not be used for measurement of directional wave spectra. For this latter application, long arrays of between 8 and 32 elements, in groups of 8 , can be built using an Octopus transceiver, supported by up to three OctRec cards, all of which are time-locked to the master clock on the Octopus card.

\section{COMPONENTS OF HF RADAR}

The DOA processing is a monostatic model, which typically has 4-elements designed for space-limited applications where large receive arrays could not be deployed. This system contains transmit antenna 4-element monopole array with sidelobe control and landward null, receive antenna beamforming array of 4 -elements, receive element single-frequency system (resonant monopole), radar receiver Quadrapus, radar Pre-Amplifier Filter (PAF) box with 4-channel pre-amplifier (configurable with up to 8 narrowband or broadband filters for each channel if multi-frequency option is desired), multi-frequency option wideband transmit antenna and loop receive antennas for 3-30 $\mathrm{MHz}$ range.

The Phased Array of Multi-static HF Radar contains transmit antenna 2-element monopole array, receive antenna beam formed by 8 -elements array of, receive element with single frequency system (reduced-size loop), radar receiver Octopus and 8-channel radar PAF box.

Bistatic DOA model is a 2 or 3 site bistatic radar where all signal reception and processing is done at the master site, with remote sites used for transmission only. Unambiguous target tracks and vector velocities are determined at the master site, using echoes received, master monostatic transmission and one or two bistatic signal echoes from the radar remote sites. Control of the remote sites is achieved by radio networking protocol and remote PC control. It contains transmit antenna 4-element monopole array with sidelobe control and landward null, receive antenna beamforming array of 4 elements, receive element single-frequency system (resonant monopole), radar receiver ISR Quadrapus, 4-channel PAF box with configurable up to 8 narrow bands or broadband filters for each channel of multi-frequency option wideband transmit antenna (loop receive antennas for 3-30 $\mathrm{MHz}$ range) $[2,3,4,5]$.

\section{NETWORKS OF HF RADAR}

Each of the above models can be configured to operate as part of a multi-static network of radars, as each card has access to Universal Time (UT) on board using a GPS receiver as part of the basic system. Both Differential GPS and RSAS capabilities are available as added options, primarily for other mobile radar applications. Under this networked approach, each radar operates within its own time slot. Thus, a twosited phased array radar would operate using alternate pulse time slots, so as to allow interleaved operation using the 
same radar frequency. Planned options include using such a pair to receive each other bistatic signal echoes as well, thus mixing monostatic and bistatic operations at both sites. This is useful for target classification and current shear measure, as is described elsewhere on these web sites:

https://tel.archives-ouvertes.fr/tel-01312183/document; http://cdn.intechweb.org/pdfs/6891.pdf; http://www.imd.gov. in/section/dwr/dynamic/radarfaq.pdf, etc.

Each model also can be configured with a multi-frequency option, requiring loop receive and multi-frequency transmit antenna or also as desired to be configured as a system to additional requirements. The system also offers a Rubidium clock option, which provides extremely low phase noise, important for high dynamic range applications, such as sea state monitoring using 2nd-order Doppler echoes or for target (ship) detecting, classification and tracking.

\section{TYPES OF HF RADAR TRANSCEIVERS}

The Octopus transceiver card family presents a state of the art digital radar capability that forms the basis of the system. It utilizes the miniaturization of RF components in the design of new radar capabilities and provides transmit and receive capability on one PCI card. It offers programmable pulse generation capability with 1 to $100 \mathrm{MHz}$ bandwidth programmability, and eight digital receiver channels using 8-bit A/D converters for the receiver section for the first step of acquisition, followed by additional onboard processing that increases the dynamic range of the recorded signal.

The single Octopus transceiver supports an 8-element receive array and can be used with up to three additional OctalRec receiver cards to increase the receive aperture to as many as 32 elements. Receiver output data are two-byte words, achieved by either repetitive pulse averaging or digital downconversion I/Q data generation. In the case of averaging, up to 256 consecutive echoes can be averaged, providing 2-byte output real data. For Digital Down Converter (DDC) processing, the gain is the ratio of $95 \mathrm{MHz}$ divided by the desired output rate ( $\sim$ pulse bandwidth). For an 8 -ms pulse, $125 \mathrm{KHz}$ bandwidth, a gain of $95 / .125=760$ is achieved, with I/Q pairs generated and stored at 4-bytes output format. On-board averaging is also available to more fully utilize the 4-byte output word size.

The waveform generation capability ranges from a few $\mathrm{KHz}$ to $100 \mathrm{MHz}$, with user-defined programmable pulse envelope control, in addition to ISR provided cosine-squared, square wave, and triangle envelopes. The signals are generated using Direct Digital Synthesizer chips with a square waveform, fed to a mixer with a user-defined pulse envelope, resulting in a pulse waveform with RF spectrum characteristics that will satisfy HF-band requirements. Software graphical-userinterface is provided that allows the user to program the transceiver according to the desired specifications for the application at hand, stores the parameter file for future use, and then executes the acquisition.

The program can be run on a periodic basis using a task scheduler for continuous data collection. The Octopus card currently operates under Microsoft Windows and Linux platforms and the Octopus transceiver fits in standard full-sized Peripheral Component Interconnect (PCI) slot (full height and length card) utilizing components of PLX Technology with a PCI9054 chip to communicate with the PCI bus. The Quadrapus transceiver is 4-channel version of the Octopus, designed for use with DOA Radar or four (fewer) receive antennas with other identical characteristics to the Octopus.

The OctRec receiver card is basically the same design as the Octopus transmitter, but without the transmitter capability. It is designed for use in larger phased array radars with 16 to 32 elements. One or more OctRec receiver cards are then used in tandem with an Octopus transceiver, which generates master A/D timing for the OctalRec cards. In the future, it will offer other passive sensing systems based on the OctalRec digital receiver. In such a way, the QuadRec receiver card is basically a Quadrapus card without the transmitter capabilities $[2,3,4,6]$.

\section{SHIP CLASSIFICATION USING MULTI- FREQUENCY HF RADAR}

The ability to operate multi-frequency HF radar allows one to classify ships and small boats. One makes use of the radar frequency dependence of the echo strength or Radar Cross Section (RCS) of individual ships. This occurs because for vertical polarization at HF frequencies, the echo strength is typically dominated by vertical structures of the order of a quarter radar wavelength, such as masts, antennas and stowed fishing lines, so in the case of large ships, the bow and stern vertical rise from the water. Use of the $3-30 \mathrm{MHz}$ range encompasses quarter wavelength ranges between 2.5 to 25 meters in height.

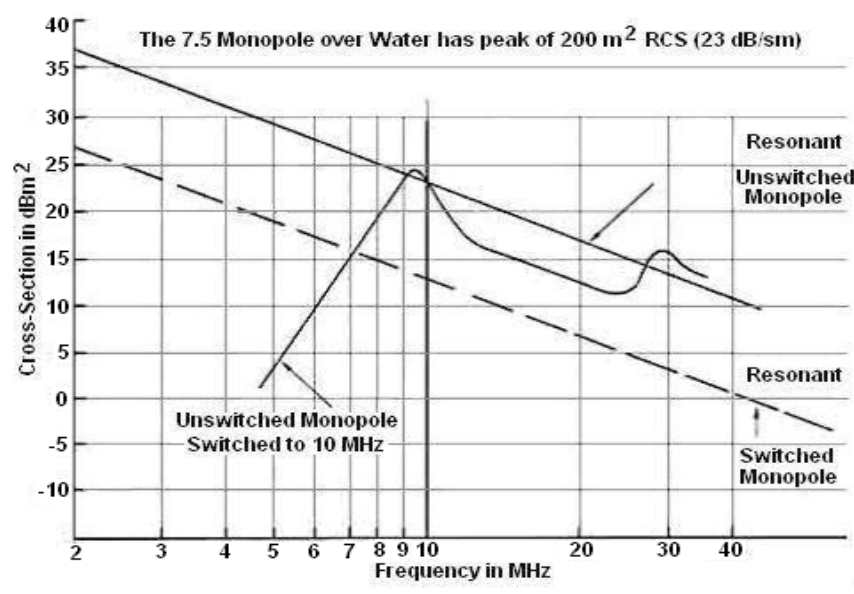

Fig. 3. Radar Measurements of Small Boats - Courtesy of Paper: by Trizna [7]

The additional use of bistatic illumination, where a second (or more) transmitter is placed a few tens of miles from the receiver site, either on land or on an offshore platform and ship at sea, adds an additional dimension to the RCS dependence. When two or more vertical structures are illuminated using a bistatic geometry, they can produce RCS maxima and 
minima that vary with bistatic aspect angle, as well as ship aspect or heading, relative to the receive site. The first figure below shows the radar cross-section frequency dependence of an ungrounded vertical mast 7.5 meters high. The shape of the region around the maximum is based on a mast width of about $10-\mathrm{cm}$ and will narrow/broaden with smaller/larger diameter masts. The solid line intersecting the Y-axis at $\sim 37 \mathrm{dBm} 2$ represents the locus along which this curve is slid for taller or shorter masts of the one-quarter length of the radar wavelength. More than one mast, or a tall radio antenna and a mast, can be considered as a pair of monopoles of corresponding lengths, and the RCS of the combination is a function of their spacing and aspect relative to the radar transmitter and receiver. Radar Measurements are done using the radar cross-section for a small boat, illustrated in Figure 3 and is seen to be well represented by a monopole resonant at 16.6 meters length, plus some additional monopole elements that are responsible for the other peaks.

Radar Modeling is a design in which two masts resonant at 8 and $12 \mathrm{MHz}$, separated by 7.5 meters were used to calculate the RCS as a function of bistatic angle for a second transmitter. The ships heading is toward the receive antenna. The effects of the changing illumination angle for coherent addition of the scatter from the two monopoles is rather complex, but can be used to classify targets using such models $[2,3,4,7]$.

\section{COASTAL HF RADAR SURVEILLANCE OF PIRATE BOATS}

The deployment of a network of HFSWR systems is a complex task with many factors to be considered, particularly when the radars are expected to perform multiple roles. Failure to treat the sitting problem with appropriate care could seriously degrade performance in one or more radar missions. Here is described a practical technique for HFSWR network design, based to the multi-objective optimization, which has to demonstrate its efficacy in the context of a hypothetical two-radar system deployed in the Strait of Malacca and off Somalia Coast, a major waterway along which many critical surveillance requirements for tracking of pirate boats have been identified. The results confirm that quite disparate criteria can be taken into account with this research approach and that this methodology can be extended to higher and successful dimensions.

\section{SYSTEM ARCHITECTURE FOR ANTI PIRACY MISSION IN STRAIT OF MALACCA}

The HFSWR surveillance network is a highly cost-effective remote sensing technology for measuring waves and currents including monitoring the movements of ships and aircraft, at over-the-horizon ranges. To place this in a specific context, the nominal performance of two representative HFSWR systems, such as the Low-Cost Civilian Radar and a more sophisticated Military Radar are summarized in Table $\mathbf{1 .}$

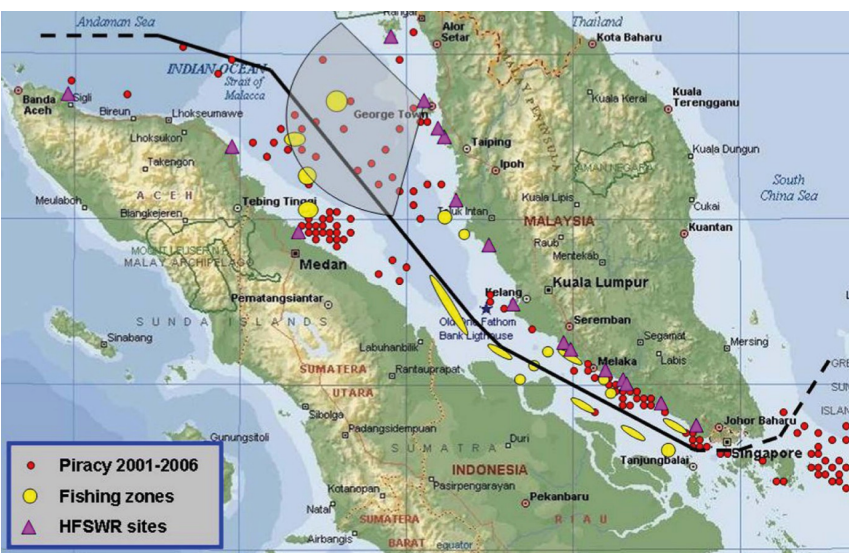

Fig. 4. Radar HF Surveillance and Remote Sensing of Pirate Ships - Courtesy of Paper: by Anderson [8]

Tab. 1. Performances of Civilian and Military Systems

\begin{tabular}{l|l|l|l|l}
\multirow{2}{*}{ Observable } & \multicolumn{4}{|c}{ Typical Performance } \\
\cline { 2 - 5 } & \multicolumn{3}{|c}{ Low-cost Civilian Radar } & \multicolumn{2}{c}{ Military Radar } \\
\cline { 2 - 5 } & Max, Range $(\mathrm{Km})$ & \pm Accuracy & Max, Range $(\mathrm{Km})$ & \pm Accuracy \\
\hline Surface Current & $60-200$ & $0.02-0.20 \mathrm{~m} / \mathrm{s}$ & $350-450$ & $0.02-0.10 \mathrm{~m} / \mathrm{s}$ \\
\hline Wave Height & $30-100$ & $10-25 \%$ & $150-259$ & $10-20 \%$ \\
\hline Wind Direction & $50-150$ & $30^{\circ}-60^{\circ}$ & $320-400$ & $20^{\circ}-60^{\circ}$ \\
\hline Wind Speed & $30-100$ & $20 \%$ & $200-250$ & $20 \%$ \\
\hline Large Ships & $50-180$ & $0.5-3 \mathrm{Km}$ & $300-450$ & $0.5-3 \mathrm{Km}$ \\
\hline Fishing Boats & $20-65$ & $0.5-2 \mathrm{Km}$ & $120-280$ & $0.5-2 \mathrm{Km}$ \\
\hline Small Ships & $10-25$ & $0.5-1 \mathrm{Km}$ & $70-150$ & $0.5-1 \mathrm{Km}$
\end{tabular}

In the above table, normal performance of representative HFSWR systems against generic mission is presented. It is noted that coverage and accuracy are dependent on sea conditions, target behaviour and other factors, consideration that is indicated here by citing the range within, in which each parameter usually lays. Figure 4 is shown the primary shipping channel in the Strait of Malacca covered by the HFSWR system, along with the locations of the main fishing zones and the areas with the highest incidence of piracy. To provide prospective coverage, the HFSWR system is deployed at a coastal radar located in George Town on Pulau Pinang Island in the Strait of Malacca. No other sensor possesses the same combination of over-the-horizon radar coverage, daynight operation with ability to detect non-cooperating targets, remote sensing of sea conditions, ships and low cost per unit area under surveillance. While some HFSWR systems have been deployed with a single mission in mind, it is increasingly recognized that the versatility of this technology supports a variety of applications.

For instance, one might wish to detect and track shipping and pirate boats in the Strait of Malacca but also to measure surface currents so that risks of collision or grounding can be minimized and any transport of pollution predicted. The most important task of anti-piracy mission in the future will be to prevent the piracy activities by deploying additional HFSWR systems in Strait of Malacca and insure more security and safety of ships and crews onboard. Accordingly, one must resort to numerical optimization techniques in a multidimensional parameter space if one is to deploy a radar to best advantage. Moreover, a single surveillance radar can 
measure only one component of the instantaneous velocity vector of a moving target. At this point, for detecting and tracking of ships and aircraft, observing target motion over time removes this limitation and the full velocity vector can be estimated

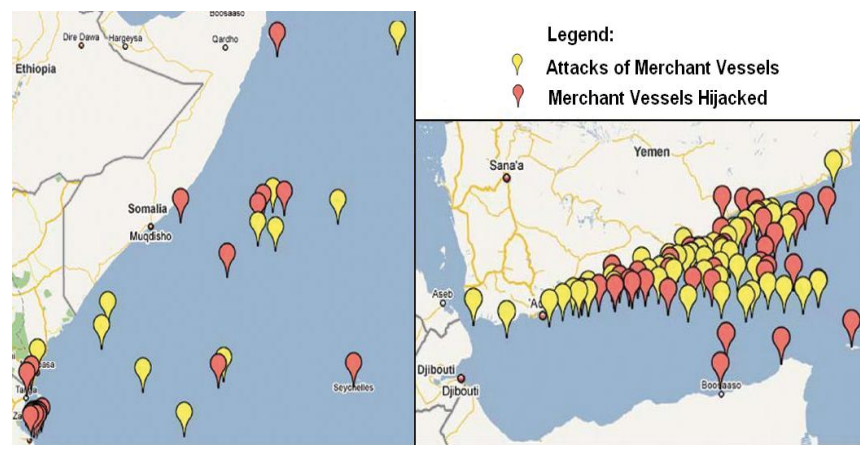

Fig. 5. Reported Piracy Incidents at Somalia Coast and the Gulf of AdenCourtesy of Booklet: by OCIMF [9]

\section{DEPLOYMENT OF HFSWR FOR ANTI PIRACY MISSION OF THE SOMALI AND ADEN COASTS}

The navigational dangers of collision and grounding of ships in the area of Somalia and the Gulf of Aden are serious problems for a long time. Armed attacks on merchant vessels transiting this area have increased in frequency over the past years. In fact, there were 140 approaches and at least 39 vessels actually detained by pirates in 2008 by source of The UK Maritime Trade Operations (UKMTO). Such acts have usually been conducted with the use or threat of violence, which can be particularly traumatic for those directly involved, as well as their families.

The Oil Companies International Marine Forum (OCIMF) is a voluntary association of oil companies having an interest in the shipment and terminalling of crude oil and oil products, which mission is to organize with its membership on matters relating to the safe shipment of crude oil and oil products, including marine pollution and safety. The Terminal Operators (SIGTTO) have an initiative with the aim of providing practical information to assist seafarers faced with potential or actual acts of piracy operating in the waters of Somalia and Aden.

Figure 5 (Left) shows s diagram of area to the South of the Horn of Africa, which is associated with Somali piracy in 2008 with events of 9 attacks on merchant's vessels, of which 8 were fired at and 10 merchant vessels hijacked.

Figure 5 (Right) shows a diagram in the area Gulf of Aden with the total number of reported 92 incidents by pirate boats. During 2008 in this area pirates organized 60 attacks on merchant's vessels, 31 vessels were fired at and 32 merchant vessels hijacked.

This High-Risk Area covers the ocean waters where attacks were frequent during past years, so to prevent actions of pirates in this area a minimum of two HFSWR installations have to be deployed, one at Somalian coast and another in the Gulf of Aden $[2,3,4,8]$.

\section{COASTAL HF JINDALEE OPERATIONAL RADAR NETWORK (JORN)}

Jindalee was the first such project taken up in the 1960s as a bistatic OTHR system controlled from the Jindalee Facility at Alice Spring (JFAS) in central Australia, where two separate transmitter and receiver sites were deployed. The main OTHR transmitter was located at Harts Range and the receiver was located at Mount Everard. However, the other radar system is an ionospheric sounder known as the Frequency Management System (FMS).

The JORN HF Radar is the OTHR network that can monitor mobile movements at sea and in the air across $37,000 \mathrm{Km}^{2}$ and it has an official range of $3,000 \mathrm{Km}$. It is used in the defence of Australia and can also monitor maritime operations, wave heights and wind directions. On 2 April 2003, Jindalee was joined with two other OTHR systems and formed what is today known as JORN system. These three radars are dispersed across Australia, at Longreach in Queensland, Laverton in Western Australia and Alice Springs in the Northern Territory, to provide surveillance coverage of Australia's Northern approaches, as shown in Figure 6 (A). This figure depicts the locations of the three OTHR systems and the JCC, and highlights the coverage of all three surveillance radars. The Alice Springs and Longreach radars cover an arc of $90^{\circ}$ each, whereas the Laverton OTHR coverage area extends through $180^{\circ}$.

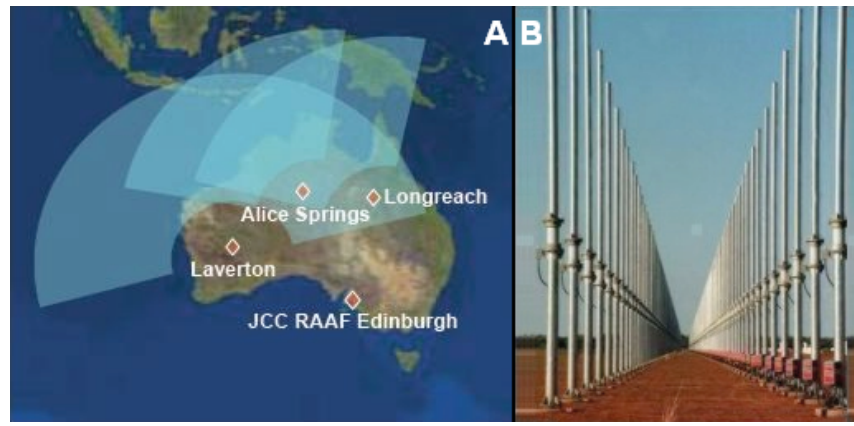

Fig. 6. Coverage Areas and Receiving Antenna of JORN - Courtesy of Paper: by Air Force/Saun [10/11]

Radar data from these three sensors is conveyed to the JORN Coordination Centre (JCC) within the Air Force's No 1 Radar Surveillance Unit (1RSU) at Royal Australian Air Force's (RAAF) Base Edinburgh in South Australia. The 1RSU site is tasked by higher headquarters to operate the JORN capability on a daily basis.

The OTHR systems operate on the Doppler principle, where an object can be detected if its motion toward or away from the radar is different from the movement of its surroundings. They are typically made up of a very large fixed transmitter and receiver antennas called arrays, which receiving antenna is illustrated in Figure 6 (B). The location and orientation of these arrays determine the lateral limits or arc of radar's coverage. The extent of OTHR coverage in range within this arc is variable and principally dependent on the state of the ionosphere. The OTHR systems do not 
continually "sweep" an area like conventional radars but rather "dwell" by focusing the radar's energy on a particular area, referred to as a "tile" within the total area of coverage. The transmitted HF energy can be electronically steered to illuminate other "tiles" within the OTHR networks coverage as required to satisfy operational tasking or in response to intelligence inquire. A disadvantage of this system is that it can detect metal vessels, while it is improbable that an OTHR will detect wooden boats. In such a way, the JORN system cannot be used for tracking small wooden boats of pirates $[3,10,11]$.

The combination of these three systems, JORN, can detect all sea and air Doppler moving targets in the area between 1,000 and 3,000 Km North of the radar sites. It is also the key component of the sea-air gap surveillance system in Australia by ensuring a surveillance area with an arc of almost $180^{\circ}$ wide and out to $2,000 \mathrm{Km}$ from the Australian coastline. The detailed characteristics of JORN transmit antenna can be found in Figures 7 (A), which shows the transmission antenna array of JORN near Longreach, in Queensland. Figure 7 (B) demonstrates an interior view of the JORN Co-ordination centre at RAAF Edinburgh, South Australia.

All radar surveillance systems normally have a problem with the bend of the Earth surface. The maximum range of the detecting radars is limited by the radio horizon, which is slightly far away than the optical horizon. The early HF communication systems have made use of ionospheric refraction to obtain spectacularly long ranges for most of the twentieth century.

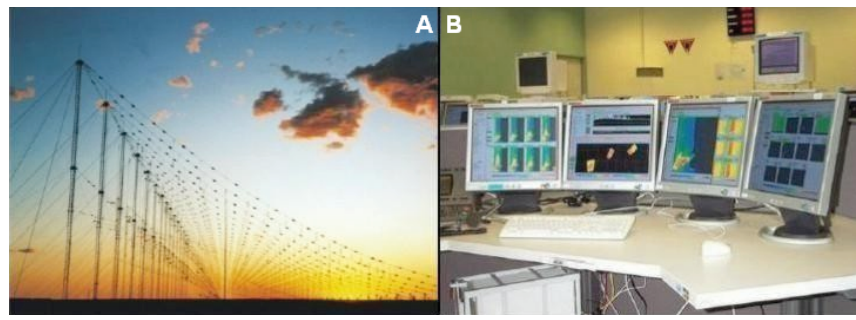

Fig. 7. JORN Transmit Antenna and Coordination Centre - Courtesy of Thesis: by Liu [12]

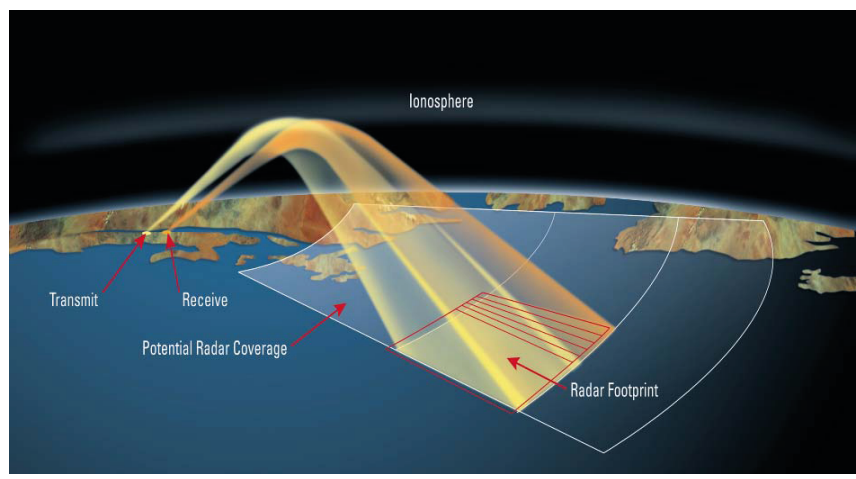

Fig. 8. Key JORN Operating Principles - Courtesy of Paper: by JORN [10]

The idea of actually radiating via the ionosphere and detecting the backscatter returning by the same path did not seem credible until coherent processing became practical in the early 1960s. Figure 8 presents the key JORN operating principles to transmit signals on $\mathrm{HF}$ frequency via ionosphere towards radar coverage and to receive reflected signals. The ionosphere is the upper part of the atmosphere extending from 75 to $450 \mathrm{Km}$ above the Earth's surface that consists of particles that have been ionized by solar radiation emitted by the Sun. Thus, the state of the ionosphere depends on the level of solar activity. Other more localized phenomena also affect the stability and/or structure of the ionosphere, which are a combination of these phenomena and solar events, which determines the quality of ionosphere support for HFSWR or OTHR operations $[3,10,12]$.

Therefore, the ability of JORN remote sensing HF radar is to see beyond the horizon with a range that dwarfed conventional radar made it an invaluable tool for detecting seagoing ships. The HF Radar is able to send signals via ionosphere towards its radar footprint or to potential radar coverage and receive reflected signals from targets at footprint and from ionosphere.

Figure 9 illustrates a diagram of the International Amateur Radio Union (IARU) that Transmit Site sends the HF signal to the ionosphere and after reflected signal from ionosphere goes to the targets, such as ships and aircraft. In the opposite direction, HF radar detects signals in Receive Site reflected from both targets via ionosphere, which finally have to be processed in Control Site [13].

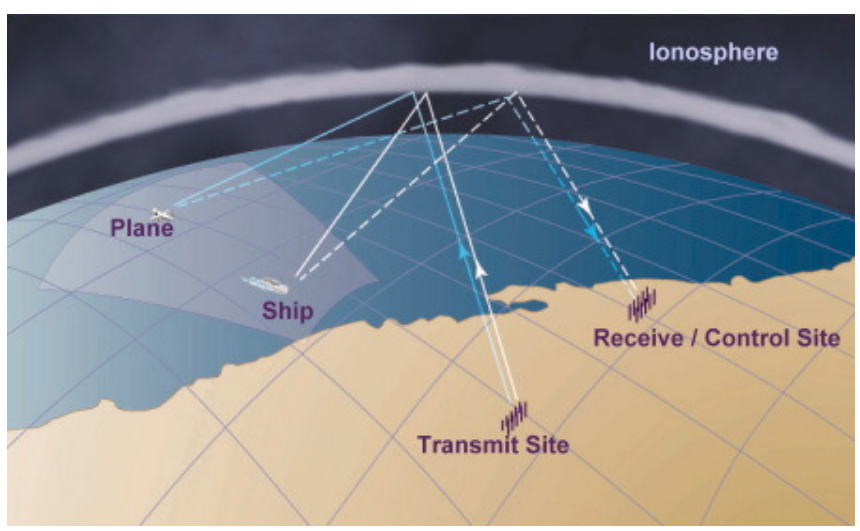

Fig. 9. Transceiver/Control Sites of HF Radar - Courtesy of Paper: by Hadel [13]

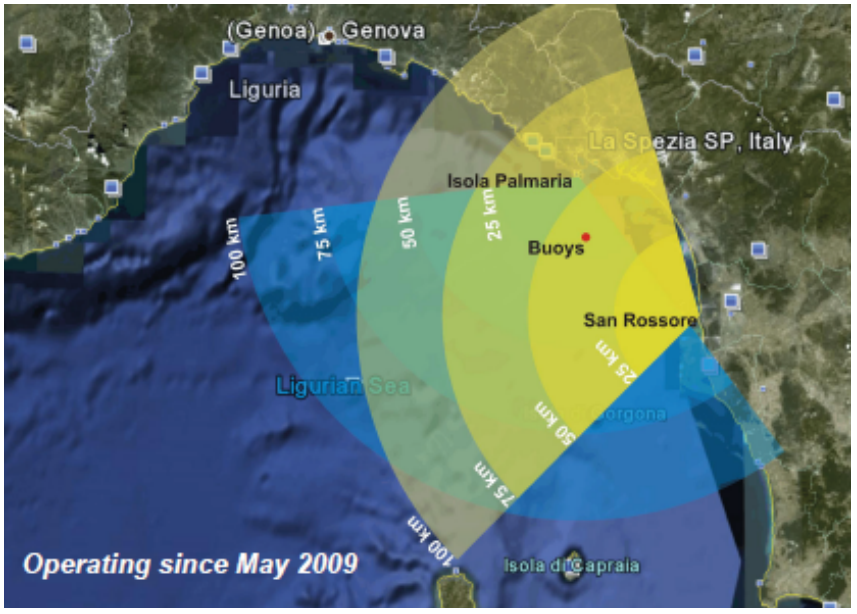

Fig. 10. Wellen Radar in Ligurian Sea - Courtesy of Paper: by Schlick [14] 


\section{DIFFERENT PROJECTS OF COASTAL HF RADARS FOR VESSELS DETECTING}

The HF surface wave radars have been identified to be a gap-filling technology for maritime domain awareness as a key factor in coastal activities for detecting and tracking of vessels in deep-sea and coastal navigation, for enhanced safety and security, improved national security (terrorism, drug smuggling, etc.) and environmental sea protection (marine protected areas, fishery monitoring and regulation, oil spills, etc.). Therefore, the HF surveillance radar is a strong candidate to become a component of any large sea area for vessel-monitoring network. This research realized several design options for an HF radar and ship monitoring system, such as multiple or single frequency, multiple or single sites or method of target bearing determination, such as Multiple Signal Classification (MUSIC) or real aperture antenna. It also presents a model of the SNR for ship detecting by HF radar and as well as observational examples of ship detecting with single and multifrequency HF radars. Finally, it suggests future experiments about tracking of pirate ships and draws conclusions. The further discussion has to estimate the primary metrics for assessing performance for ship tracking radars with Probabilities of detecting (Pd) and false alarm (Pfa) including their variation with parameters such as range, azimuth and frequency, and number of observing modes.

There are many different projects of Coastal HF Radars for detecting and tracking seagoing ships, but here will be introduced the network coverages of the German Wellen Radar (WERA) and the US SeaSonde.

1. WERA HF Radar - This type of HF coastal surveillance radar was employed in May 2009 in an international project known as "NURC BP09 Experiment" by the NATO Research Centre (NURC) of La Spezia, Italy, Department of Information Engineering, University of Pisa, Italy and Institute of Oceanography, University of Hamburg, Germany. The NURC effective maritime situational awareness project deployed a Maritime Surveillance System (MSS) that includes vessel ground truth and contact simulation, sensor processing, data fusion, anomaly detecting, sensor management and performance evaluation. Two WERA systems operating at $12.5 \mathrm{MHz}$ have been installed at the Italian coast for experimental and evaluation purpose.

Figure 10 illustrates a map depicting the HF setup in the Ligurian Sea near La Spezia town in Italy. The red dot shows the location of a Meteo and a wave rider buoy, which were deployed for validation purposes. The Ligurian Sea setup is depicted showing the overlap area in which the current and wave retrieval is performed as well as the range.

The WERA HF radar system transmits an average power of $30 \mathrm{~W}$ but it achieves detecting ranges up to $200 \mathrm{Km}$, which are far beyond the conventional microwave radar coverage. Due to external noise, radio frequency interference and different kinds of clutter, special techniques of target detecting such as ships using the WERA system have to be applied. For a 12-hour period, HF radar data have been recorded and processed. The target locations detected by the HF radar using the proposed adaptive technique are passed to a tracking filter to track the ship position. In order to estimate the performance of the radar detecting and tracking techniques, these ship locations are compared with the ship positions recorded by the VHF AIS.

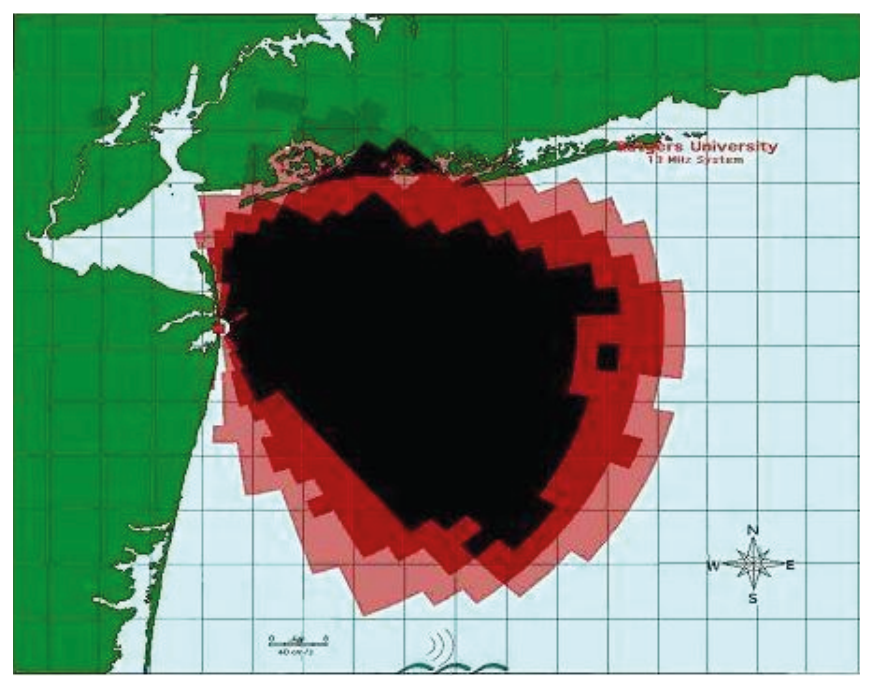

Fig. 11. SeaSonde Radial Coverage Map - Courtesy of Paper: by Glen [15]

The WERA surveillance network was developed at the German University of Hamburg in 1996 to allow a wide range of working frequencies, spatial resolution and antenna configurations in order to operate as low power oceanographic radar providing simultaneous wide-area measurements of surface currents, ocean waves, wind parameters and detecting of ships. This HF Radar system is based on a modular design that can be easily adapted to the requirements of an actual application.

2. SeaSonde HF Radar - Present SeaSonde HF radars have been designed to map surface currents, but are able to track surface vessels in a dual-use mode. The US Rutgers University in New Jersey and CODAR Ocean Sensors from Mountain in California, have collaborated on the development of vessel detecting and tracking capabilities from the compact HF radars, demonstrating that ships can be detected and tracked by multistatic HF radar in a multiship environment while simultaneously mapping ocean currents.

Furthermore, the same vessel is seen simultaneously by the radar-based on different processing parameters, mitigating the need to preselect a fixed set and thereby improving detecting performance. The radar was deployed in Sea Bright, New Jersey, $40 \mathrm{Km}$ south of the Battery in New York City. It was direction-finding type radar, SeaSonde Remote Unit code SSRS-100, manufactured by CODAR Ocean Sensors and was installed in October 2008. The radar's primary function was the measurement of surface currents, which are provided in real-time to the NOAA National HF Radar Network. The radar also has the dual-use capability to detect the location of ships at sea.

Figure 11 illustrates the spatial and temporal radial vector coverage for ocean currents of the radar over a 1-week period, 
which coincided with the ship detecting exercise. The radial coverage map is presented in the area of the SeaSonde at Sea Bright, New Jersey, over a 1-week period. The color map illustrates the temporal coverage along the radial grid (black $=75 \%$, red $=50 \%$ and pink $=25 \%$ ). The radar collected range data, which are a time series of the complex echo signal voltages before Doppler processing. These range files are the result of the first Fast Fourier Transform (FFT) of the frequency-modulated continuous-wave received signal.

The range data were collected using an FFT length of 512 points. With a $2-\mathrm{Hz}$ sweep of the radar, each range file encompasses $256 \mathrm{sec}$ of coherent integration time. There were a total of 15 range files over the hour-long period. However, the time on the computer and all the subsequent files it generates are synchronized to atomic time via GPS by the Macintosh operating system. The SeaSonde HF radar consists of a compact receive antenna with three elements, such as two-directional crossed loops and an omnidirectional monopole, a monopole transmit antenna and hardware housed within a climate-controlled enclosure. The radar transmits a radio wave with a centre frequency of $13.46 \mathrm{MHz}$ and a bandwidth of $50 \mathrm{kHz}$. The bandwidth of the radar sets the spatial range resolution of the system, which was about $3 \mathrm{Km}$ for this particular bandwidth. Separate transmit and receive radar antennas were used for this study spaced at least one a wavelength apart, which is approximately $23 \mathrm{~m}$ at the $13 \mathrm{MHz}$ radio band. A ship with a vertical structure of a quarter wavelength $(6 \mathrm{~m})$ is the minimum-sized optimal reflector $[3,4,14,15]$.
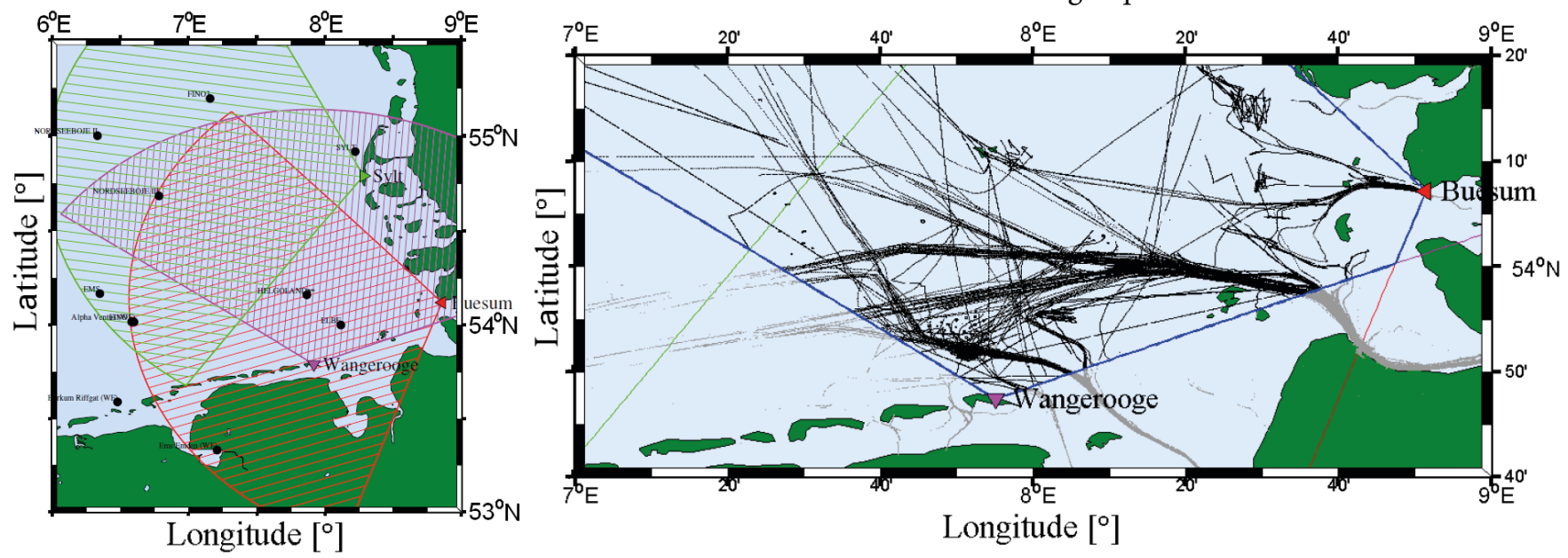

Figure 12. Setup of the HFSW radars in the German Bight and AIS Ship Routes - Courtesy of Paper: by Grasso [16]

3. German Bight HF Radar - In the context of ships surveillance, a cost-effective long-range early-warning sensor for ship detection and tracking, which provide data fusion techniques to live-recorded data from a network of oceanographic HFSW radars installed in the German Bight (North Sea), shown in Figure 12 (Left), where Sylt is green, Busum (red) and Wangerooge (magenta) are demonstrated. This experimentation closely follows the one conducted in the Ligurian Sea (Mediterranean Sea) by
NATO Science and Technology Organization (STO) Centre for Maritime Research and Experimentation (CMRE) during the Battlespace Preparation 2009 (BP09) campaign. In such a way, ship reports from the AIS data, recorded from both coastal and satellite-based stations are exploited as ground truth information and a methodology is applied to classify the fused tracks and to estimate system performances. Figure 12 (Right) illustrates the AIS contacts recorded at Busum and Wangerooge stations on 4th August 2013. A total of 620 different AIS-carrying ships (grey) were recorded that day, with 299 only in the fusion region (blue line perimeter). In fact, this number is far greater than previous conducted, and the performance assessment procedure requires that the AIS reports are interpolated (black) on the radar timestamps $[2,3,16]$

\section{CONCLUSION}

Modern radar systems design offers advanced features such as automatic tracking of vessels in coastal waters and geographical mapping. Geographical masks or areas can be introduced to define forbidden zones, speed limit zones, traffic separation zones etc. However, in order to detect and track ocean-going ships and especially small vessels trying to evade authorities, sophisticated methods of detection and tracking are required. At this point, specialized software algorithms are developed, which ensure successful tracking even in extremely difficult weather and sea conditions have to fulfil the following requirements:
Longitude $\left[^{\circ}\right]$
- High probability of detection and low false alarm rate;

- Ability to discriminate between close targets;

- Noise and clutter suppression processing techniques;

- Stable tracking and rapid manoeuvre detection;

- Analysis of target movements to identify suspicious behaviour patterns; and

- The application of predefined alarms that respond to entry into zones or in close proximity to other targets.

The coverage area of fixed coastal radar stations can be quickly recognized and the illegal traffic can be directed outside of the existing coverage areas. The radar coverage 
areas can be extended and continuously changed by use of transportable radar stations. In this way, the detection zones can be made unpredictable and hence their employment increases the probability of preventing illegal activities. The portable radar stations can be housed inside equipment containers, which can be moved around by helicopter or a lorry. If a more rapid deployment of radar stations is required, the radar sensor can be housed inside a van. Thus, in both cases the radar sensors are self-contained, having power generators, air-conditioning and radio communications.

The International Ship and Port Facility Security (ISPS) Code has been adopted and approved by the International Maritime Organization (IMO) to address the threat to the maritime industry posed by terrorism and other illegal activities. The Code was approved in December 2002 and became a law on 1st July 2004. The implementation of ISPS code calls for improved detection of small craft in littoral waters. The use of radar sensors is a primary tool in the detection of small unfriendly vessels. In most cases the high quality radar data may be the only source of information indicating the position and movements of vessels posing security threats. Therefore, a careful choice of radar parameters is very important for the detection and discrimination of small targets.

\section{REFERENCES}

1. Isik C. \& Others, "Principles of Integrated Maritime Surveillance Systems”, Kluwer, Norwell, MA, 2000.

2. Ilcev D.S., "Global Mobile Communication, Navigation and Surveillance (CNS)”, DUT, Durban 2012.

3. ISR, "HF Surface Wave Radars", Imaging Science Research (ISR), Burke, VA, 2015.

4. Fabrizio G., "High Frequency over the Horizon Radar", McGraw-Hill, New York, 2013.

5. Trizna D.B., "A Bistatic HF Radar for Current Mapping and Robust Ship Tracking”, Paper, Burke, VA, 2010.

6. Ilcev D.S., "Maritime Communication, Navigation and Surveillance (CNS)”, Presentation, DUT, Durban 2014

7. Trizna D.B. \& Xu L., "Microwave and HF Multi-Frequency Radars for Dual-Use Coastal Remote Sensing Applications”, Paper, Paper, Burke, VA, 2005.

8. Anderson S.J., "Optimizing HF Radar Siting for Surveillance and Remote Sensing in the Strait of Malacca", IEEE Transactions on Geoscience and Remote Sensing, Vol. 51, No 3, Piscataway, NJ, 2013.

9. OCIMF, "Piracy - The East Africa/Somalia Situation", Oil Companies International Marine Forum, London, 2008.
10. Air Force, "Jindalee Operational Radar Network", Fact Sheet, Canberra, Australia, 2015.

11. Saun G., “Jindalee Operational Radar Network”, Department of Defense, Edinburgh, Australia, 2015.

12. Liu B.Y., "HF Over-the-Horizon Radar System Performance Analyzes”, Thesis, Naval Postgraduate School, Monterey, CA, 2007.

13. Hadel W., "Radar Systems on Shortwave", International Amateur Radio Union (IARA), Newington, CT, 2015.

14. Schlick T. \& Others, "Evolution of a HF-Radar Ship Detection Algorithm by Comparison to AIS and SAR Data", NATO Undersea Research Center/University of Hamburg, La Spezia, Italy/Hamburg, Germany, 2011.

15. Glen S.M. \& Others, "Performance Evaluation of SeaSonde High-Frequency Radar for Vessel Detection", CODAR Ocean Sensors, Mountain View, CA, 2010.

16. Grasso R. \& Others, "A Network of HF Surface Wave Radars for Maritime Surveillance Preliminary Results in the German Bight”, NATO STO CMRE, La Spezia, Italy, 2014.

\section{CONTACT WITH THE AUTHOR}

Prof. D.S. Ilcev, BSc, MSc, PhD

e-mail: ilcev@dut.ac.za,tel.: +27827650817

Master Mariner

Chairman of Spice Science Centre (SSC) and PostgraduateStudies at:

Durban University of Technology (DUT) 45 Bencorrum, 183 Prince Street, Durban 4001

South Africa 